\title{
Intensity based optical measurement of cornea vibration
}

\author{
J. Osmers, M. Sorg, A. Fischer \\ University of Bremen, BIMAQ - Bremen Institute for Metrology, Automation and Quality Science, \\ Email: osm@bimaq.de
}

\begin{abstract}
In order to measure the dynamic behavior of eyes for yielding the intraocular pressure an optical, intensity-based sensor approach is developed. The challenge is to emit less than $1 \mathrm{~mW}$ of power to avoid harming the eye while coping with the low reflection of the eye of $4 \%$ for normal incidence. With solely analog infrared diodes and phototransistors the vibration of the cornea of an enucleated porcine eye is demonstrated to be detectable for the whole frequency range of its eigenvalues. For demonstrating the contactless measurement of the eye vibration response that goes with the pressure excitation, the cornea was coated with $\mathrm{TiO}_{2}$ powder first. An optimization of the optical arrangement that maximizes the reflected light power finally enables measurements without coating. As a result, the signal-to-noise ratio of the proposed sensor setup for contactless eye vibration measurements could be enhanced from $24 \mathrm{~dB}$ to $46 \mathrm{~dB}$. The expected resolution of the proposed sensor for the IOP measurement is calculated to $\sigma(\mathrm{IOP})<3 \mathrm{mmHg}$ in the physiological range, which gives high application potential.
\end{abstract}

Key words: tonometry, dynamic behavior of eyes, optical measurement, glaucoma

\section{Introduction}

Glaucoma is one of the major risks of blindness worldwide. It is a disease that proceeds unnoticed since it does not cause pain and initial loss of the visual field is interpolated by the human brain [1]. Glaucoma encompasses etiologically similar diseases that lead to a characteristic loss of neuronal ganglion cells [2]. The circular spot, where the neuronal cells bend into the optic nerve, called the papilla is damaged by an individually too high intraocular pressure (IOP). The IOP describes one of the major risk factors for glaucoma. Lowering the IOP is the only therapeutic approach in order to stop further damage of the papilla. So far there is no chance to cure the eye disease glaucoma or to regenerate lost neural connections. This makes diagnostic and state preserving therapy the most effective treatment [2], [3]. A reliable measurement of the IOP with low measurement uncertainty is crucial for monitoring surgical success or the effect of medical treatment.

Existing devices to measure the IOP, called tonometers, are based on Newton's third law, where the force is measured that is required to indent the front of the eye (the cornea) to a certain amount. The clinical reference therefor is the Goldmann applanation tonometer (GAT). The patient's eye has to be anesthetized in order to measure the IOP with a glass stamp that indents the cornea of the eye, so that a specified area with a diameter of $3.06 \mathrm{~mm}$ is flattened. The used force is the measurand for the IOP. The standard uncertainty of the GAT measurement is comparably low with $1-2 \mathrm{mmHg}$ [4]. Aside from the required anesthetics, the procedure is unpleasant, holds infection risks and is executable by medics only. Other contactless devices, such as air-puff tonometers do not meet the clinical requirements of a standard uncertainty of $\pm 3 \mathrm{mmHg}$ and are only used for ambulant screening. The standard uncertainty for air-puff tonometers is $\pm 5 \mathrm{mmHg}$. Due to sprayed tear fluid they also hold an infection risk.

For these reasons, a novel measurement principle for a handheld non-contact tonometer shall be validated to overcome existing disadvantages of current tonometry methods. The objective is to enable the patient to check his own IOP in the home environment. The measurement approach evaluates the dynamic reaction of the eye to a short pressure pulse generated by a loudspeaker [5], while the eye forms a coupled system with the loudspeaker and an enclosed pressure chamber. In order to detect the dynamic movement of the cornea, an optical measurement concept, which is small, light and least complex has to be developed. Former concepts to measure the cornea vibrations were based on interferometry [6], [7], [8], which is precise but too complex for the desired application. 
In order to measure the oscillation amplitude of the cornea for yielding the intraocular pressure an optical, intensity-based sensor approach is realized. The challenge is to emit less than $1 \mathrm{~mW}$ of radiation power to avoid damage to the eye. Due to the low reflection factor of the eye of $4 \%$ for normal incidence, the signal-tonoise ratio is insufficient for reliable measurements. For demonstrating the contactless measurement of the eye vibration response that goes with the pressure excitation, the cornea of enucleated porcine eyes was coated with $\mathrm{TiO}_{2}$ powder first. With an analog infrared diode and a phototransistor the vibration of the cornea is demonstrated to be detectable for the whole range of its eigenvalues. An optimization of the optical arrangement enhances the signal-to-noise ratio and finally enables measurements without coating.

\section{Methods}

Laboratory tests on porcine eyes have been realized in the conception phase as a preparation for the handheld tonometer. The measurement setup is depicted in Fig. 1. It consists of an aluminum pressure chamber to which a loudspeaker is connected and a microphone to measure the sound pressure variation. The top of the pressure chamber has a circular vent that is sealed by an enucleated porcine eye during the measurements.

Through generated pressure pulses from the loudspeaker, the eye is excited and starts to oscillate. In order to obtain detailed information about the oscillation behavior of the eye, which depends on the IOP, the cornea oscillation has to be measured directly. For avoiding influences on the measurand, an optical measurement approach is preferred. The different sensor types that could be used for this task have been rated in terms of resolution in displacement $(<10 \mu \mathrm{m})$ and time $(>5 \mathrm{kHz})$, size $(20 \mathrm{~mm} x$ $30 \mathrm{~mm} \times 20 \mathrm{~mm})$, weight $(<150 \mathrm{~g})$, prize $(<500 €)$ and eye safety $(<1 \mathrm{~mW})$. The respective sensor evaluations for a vibrometer, a triangulation sensor and a reflection sensor are summarized in table 1. According to the rating, the best suited technology is the analog reflection sensor due to its small dimensions, the light weight and the extremely low prize.
Tab. 1: Contrast table of possible optical metrological devices. The rating goes from 1 to 4 where 4 means best suited for this application. The objective is to find a sensor type that meets the requirements for a handheld battery operated device that is eye safe and affordable for patients.

\begin{tabular}{|c|c|c|c|}
\hline & Vibrometer & $\begin{array}{c}\text { Triangulation } \\
\text {-sensor }\end{array}$ & $\begin{array}{c}\text { Reflection } \\
\text { sensor }\end{array}$ \\
\hline resolution & 4 & 3 & 2 \\
\hline $\begin{array}{c}\text { frequency } \\
\text { limit }\end{array}$ & 4 & 2 & 4 \\
\hline size & 1 & 3 & 4 \\
\hline weight & 1 & 3 & 4 \\
\hline prize & 1 & 2 & 4 \\
\hline eye safety & 2 & 2 & 3 \\
\hline $\begin{array}{c}\text { Mean of } \\
\text { rates }\end{array}$ & 2.17 & 2.5 & 3.5 \\
\hline
\end{tabular}

The chosen reflection sensor consists of an infrared diode, which emits light at $950 \mathrm{~nm}$, and a phototransistor that is sensitive for this wavelength. In order to detect the movement of the cornea the sensor is placed $5 \mathrm{~mm}$ in front of the enucleated porcine eye.

Two types of measurements have been carried out. At first, the eye is placed on the vent with the cornea faced to the inside of the chamber. With this orientation, the measurements are performed perpendicular to the cornea. Due to the low reflection, the cornea has been coated with a $\mathrm{TiO}_{2}$ anti-glare spray for increasing the reflected light intensity.

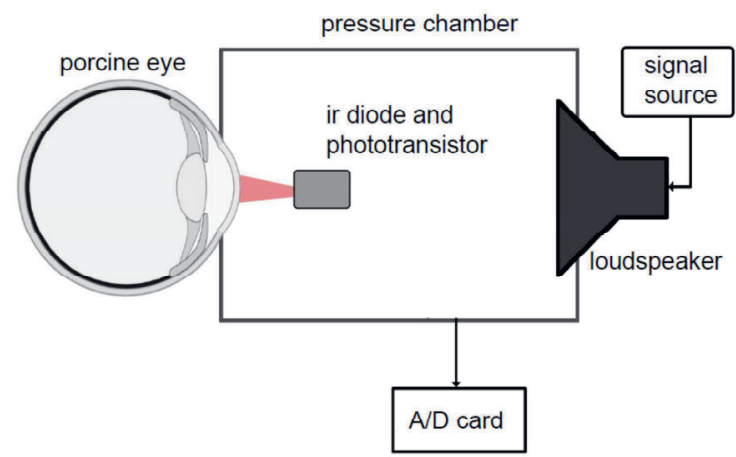

Fig. 1: Symbolic measurement setup for vibration analysis of porcine eyes, with infrared diode and phototransistor $5 \mathrm{~mm}$ in front of the cornea.

For the second measurement setup, the eye is turned around to have better accessibility with respect to a change of the angle a between the infrared diode and the phototransistor, see Fig 2. 


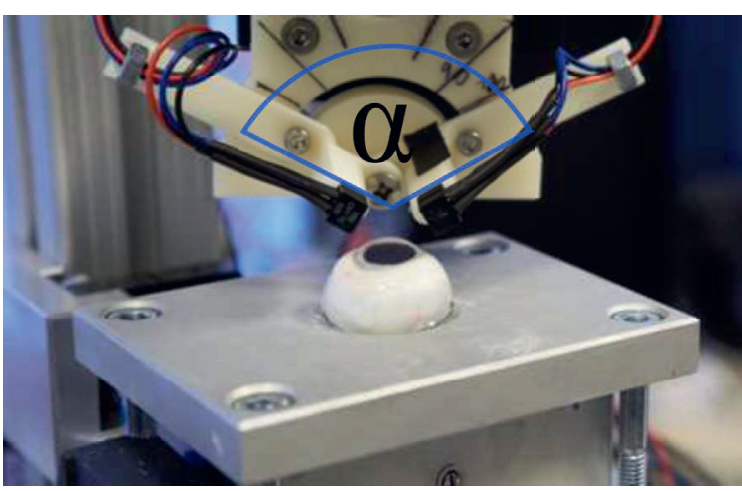

Fig. 2: Measurement setup for cornea vibration measurement with infrared reflection sensors on an enucleated porcine eye. The eye was turned around to analyze the angle dependency $\alpha$ between emitter and transistor. For the left reflection sensor only the diode and for the right sensor, only the transistor is connected.

In this orientation, the impulse travels from the backside of the eye and excites the cornea to oscillate, though the amplitude is smaller.

The vibration of the cornea modulates the light intensity of the diode that is reflected to the transistor. With an analog-to-digital card the resulting voltage difference is sampled at $51.2 \mathrm{kHz}$ and 24 Bit resolution. The excitation signal is a sinusoidal sound wave of $107 \mathrm{~Hz}$. The sound pressure in the chamber amounts to $150 \mathrm{~dB}$.

\section{Results}

Initial measurements are taken at a loudspeaker diaphragm to analyze the possible frequency resolution of the sensor. Through its analog and smooth characteristic there is no necessity for further signal conditioning, so that the signal can directly be processed by evaluation algorithms without filtering. The signal quality can be seen in Fig. 3 .

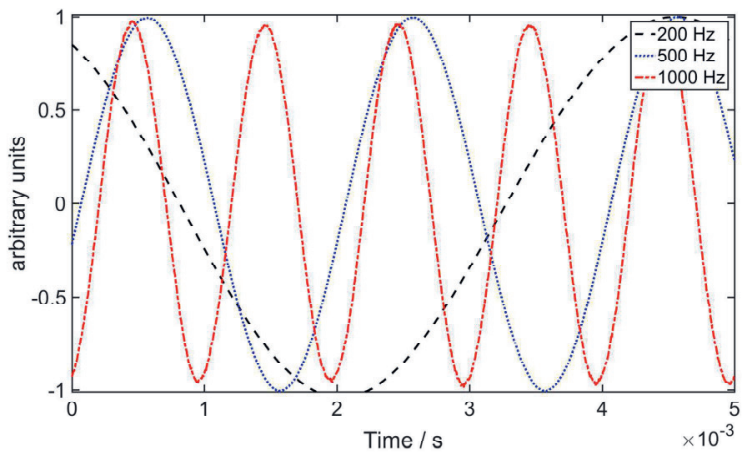

Fig. 3: Frequency resolution of reflection sensor for the movement of the speaker's diaphragm. Frequencies up to $1 \mathrm{kHz}$ can easily be detected by the sensor. No filtering was used for the presented data.

The voltage amplitude of the sensor output directly refers to the amplitude of the speaker's diaphragm. According to the electro-mechanic properties of the speaker, the oscillation amplitude decreases by the frequency, which means that the spatial and temporal resolution of the reflection sensor, are tested simultaneously. The highest frequency tested, has the lowest amplitude of a few $\mu \mathrm{m}$. Due to the normalization in Fig. 3, the signal-to-noise ratio decreases proportional to the amplitude since the standard uncertainty of the sensor signal remains constant.

It is expected from former research results that the relevant frequencies that dominate the dynamic behavior of the eye stay below $1 \mathrm{kHz}$ [6]. According to the Nyquist theorem, the resolution should be at least twice as high as the expected frequency to be measured. The preliminary test on the speaker's diaphragm shows that the sensor easily covers the desired frequency range, since all frequencies are neatly detected.

In order to enlarge the reflection of the eye, the first laboratory tests were carried out with antiglare $\mathrm{TiO}_{2}$ spray. Only for this measurement, the loudspeaker is actuated by a $20 \mathrm{~Hz}$ rectangular signal. The pressure variation in the chamber causes the eye to vibrate, cf. Fig 4. The oscillation of the cornea follows the pressure fluctuations in the chamber and shows an overlying harmonic that may result from the mechanical properties of the eye. As a result, it is now possible to measure the cornea vibrations resulting from the loudspeaker excitement. Without the coating, the detector signal is reduced by a factor of 10 to 20 , which disallowed signal acquisition.

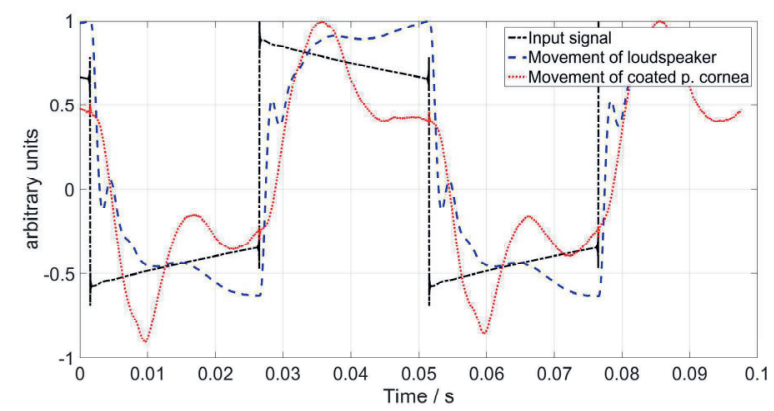

Fig 4: Rectangular excitation signal of $20 \mathrm{~Hz}$ with detected movements of the loudspeaker diaphragm and the coated porcine cornea. This measurement demonstrates the feasibility of fast vibration detection with the sensor setup shown in Fig. 2.

However, by optimizing the angle a between the infrared diode and the phototransistor, the reflected intensity from the cornea and, thus, the signal-to-noise ratio is enhanced according to the Fresnel equations. Fig. 5 shows the sinusoidal excitation signal of $107 \mathrm{~Hz}$ and the detector output for four different angles of $\alpha$. The detected amplitude of the phototransistor rises with an increasing angle $\alpha$. The oscillation 
of the cornea stays constant and the vibration can clearly be allocated to the excitation due to the same periodicity. The signal noise of the sensor is at a voltage range of $1.5 \mathrm{mV}$ which leads to a signal-to-noise ratio of $24 \mathrm{~dB}$ for $\alpha=54^{\circ} ; 40 \mathrm{~dB}\left(\alpha=90^{\circ}\right), 42 \mathrm{~dB}\left(\alpha=120^{\circ}\right)$ and $46 \mathrm{~dB}$ for $\alpha=150^{\circ}$. As a result, the measurement at uncoated porcine cornea is now possible due to an increase of $\alpha$. At higher angles the sensor touched the anterior part of the porcine eye.
The emitted power of the infrared diode was $0.018 \mu \mathrm{W}$, which is considered eye safe.

With an increase of the angle the yield of the reflected intensity rises, which also means, that the transmission decreases. Therefore the signal-to-noise ratio can possibly be optimized further since the radiated power can increase by a factor of 50 without putting the eye at risk.

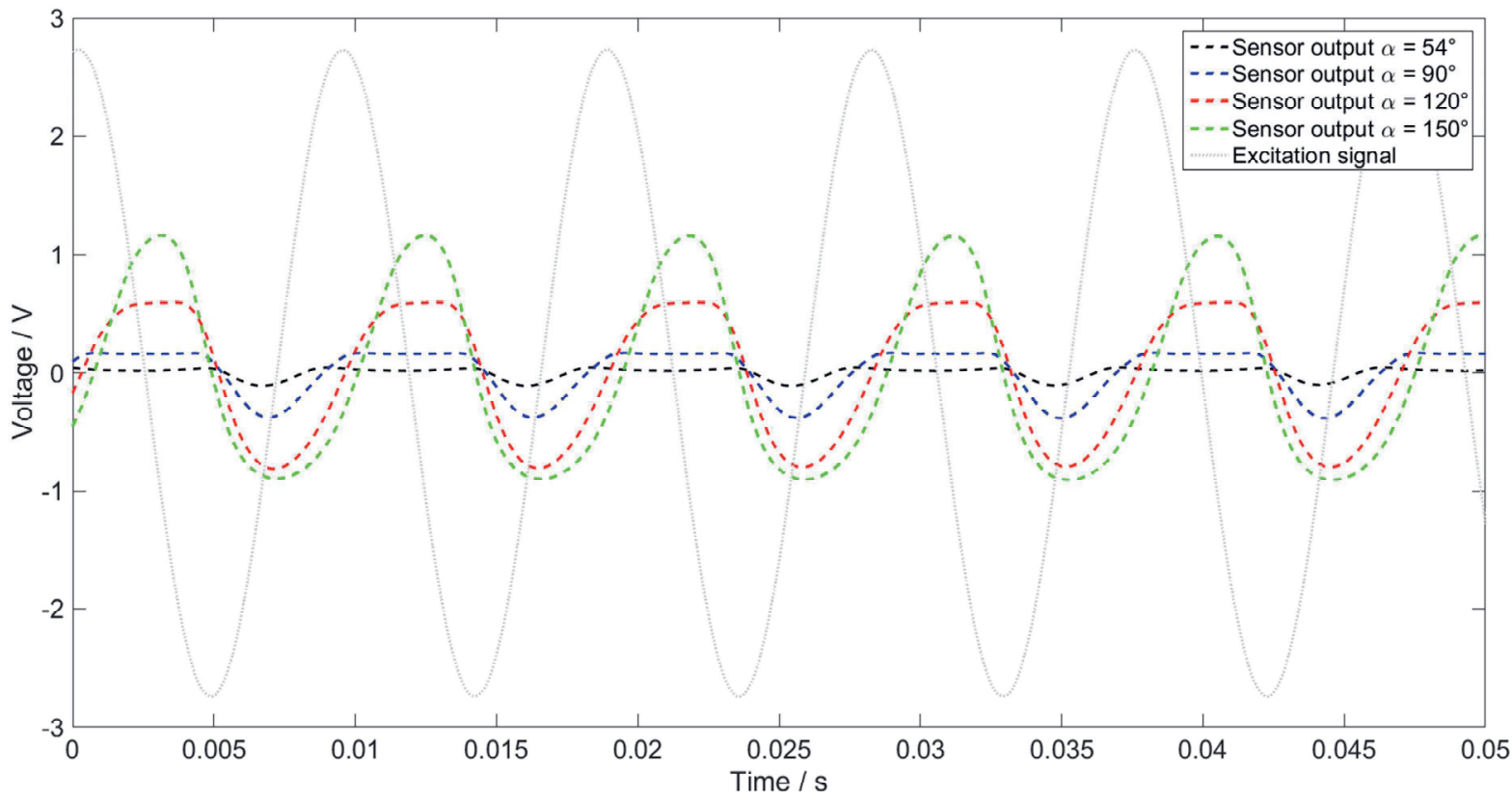

Fig. 5: Qualitative displacement of uncoated porcine cornea detected with the shown setup at 4 different angles with sinusoidal excitation at $107 \mathrm{~Hz}$. The periodicity shows the dependency from the input signal with a phase shift. The clear detector signal demonstrates the low signal-to-noise ratio of the measurement system, which grows by the angle. The vibrational response of the cornea will be evaluated to determine the intraocular pressure.

Through measurement results with a confocalchromatic sensor on porcine eyes, the quantitative amplitude of the cornea vibration can be used to estimate a possible resolution for different IOP levels with this measurement setup. Note, that the latter measurements of the quantitative cornea oscillation amplitude $A_{c}$ were performed independently with a reference sensor. According to equation 1:

$$
\sigma(\mathrm{IOP})=\frac{\partial(\mathrm{IOP})}{\partial\left(\mathrm{A}_{\mathrm{c}}\right)} \cdot \frac{\partial\left(\mathrm{A}_{\mathrm{c}}\right)}{\partial(\mathrm{U})} \sigma_{\mathrm{U}},
$$

where $\partial(\mathrm{IOP}) / \partial\left(\mathrm{A}_{\mathrm{c}}\right)$ is the derivative of the adjusted IOP and the amplitude of the cornea movement. This is multiplied by the derivative of $A_{c}$ and the measured transistor output at $\alpha=150^{\circ}$ and by the standard uncertainty of the voltage amplitude for 10 periods. The estimated standard uncertainty for the IOP measurement due to the standard uncertainty of the voltage amplitude of the phototransistor output is depicted in Fig. 6.

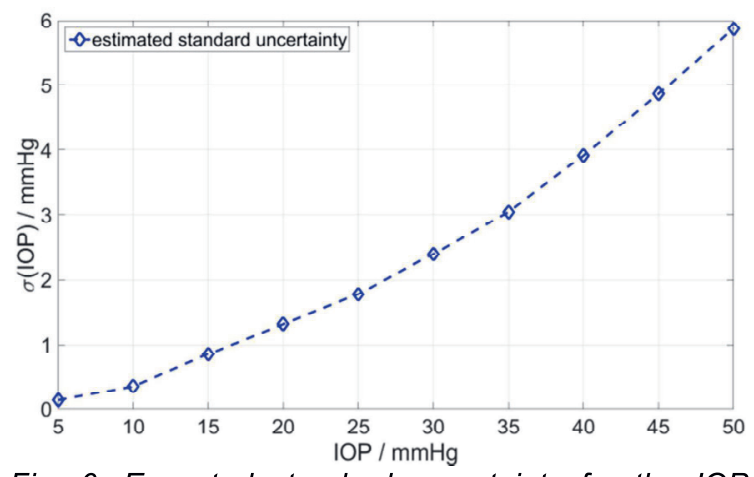

Fig. 6: Expected standard uncertainty for the IOP measurement from equation 1 at $\alpha=150^{\circ}$.

Up to $20 \mathrm{mmHg}$ the standard uncertainty is lower compared to the GAT measurement. This result already has potential for IOP estimation. The estimated standard uncertainty can probably decrease by raising the emitter current. Future research will focus on the calibration of the sensor to reach a quantitative result. 
Further challenges remain that require discussion. First, due to the intensity based measurement approach, the sensor needs to be close $(<10 \mathrm{~mm})$ to the specimen, because the intensity declines by the square of the distance. Therefore the characteristic of the displacement function also shows the highest sensitivity close to the specimen. Note further that the convex shape of the cornea scatters the reflected light. This requires a sufficiently small radiation angle $\left(<10^{\circ}\right)$ of the diode so that most of the emitted radiation is reflected onto the photo sensitive area of the transistor.

The second challenge in order to calibrate the measurement device is that a change in reflection factor of the specimen influences the sensor output signal. As a result, a change of the distance or the reflectivity of the specimen cannot be distinguished in the measurement. This reveals the cross-sensitivity of the measurement principle. This problem is solvable by using a combination of the shown setup with a slow displacement reference sensor that measures the mean distance to the specimen. The reflection sensor is then mounted at the same distance and can now detect the fast cornea oscillation with the correct distance and sensitivity. The signal can be corrected by the measured displacement in case there is a variation of the average displacement. Furthermore it can be assumed for most objects to be tested, that the reflection factor stays constant during the measurement.

\section{Conclusion}

In order to achieve a low volume, weight and prize sensor that is able to detect oscillations of the cornea, the analog reflection sensor which fitted the requirements best and actually produced feasible results. Further research will focus on the calibration of the sensor in order to achieve quantitative amplitude values that are proportional to the IOP. With required adaptations the concept could be used to measure the cornea oscillation responding to pressure excitation. The expected resolution shows the applicational potential of the shown approach, which can presumably be enhanced due to power reserves. This helps for a novel tonometry method that is gentle and solves major disadvantages of current principles.

\section{Acknowledgments}

The authors would like to thank the German Ministry of Education and Research for funding the project SelTon (13GW0054).

\section{Literaturverzeichnis}

[1] J. A. Giaconi, S. K. Law, K. NouriMahdavi, A. L. Coleman, J. Caprioli, "Pearls of Glaucoma Management", 2016.

[2] F. Grehn, Augenheilkunde, SpringerVerlag, 2013.

[3] E. Yung, V. Trubnik und L. J. Katz, „An overview of home tonometry and telemetry for intraocular pressure monitoring in humans," Graefe's Archive for Clinical and Experimental Ophthalmology, Bd. 252, pp. 11791188, 2014.

[4] A. Rosentreter, M. Neuburger, J. F. Jordan, A. M. Schild und T. S. Dietlein, „Einflussgrößen auf die Applanationstonometrie--ein praxisorientierter Überblick," Bd. 228, C. Erb, Hrsg., Georg Thieme Verlag KG Stuttgart New York, 2011, pp. 109-113.

[5] A. von Freyberg, M. Sorg, M. Fuhrmann, C. F. Kreiner, J. Pfannkuche, T. Klink, D. Hensler, F. Grehn und G. Goch, „Acoustic tonometry: feasibility study of a new principle of intraocular pressure measurement," Journal of glaucoma, Bd. 18, pp. 316-320, 2009.

[6] J. Drescher, „Bestimmung des Intraoculardrucks aus dem Schwingungsverhalten des Humanauges," 2000.

[7] A. Gundlach, „Interferometrische Schwingungsanalyse des menschlichen Auges zur Bestimmung des Intraokulardrucks," 2003.

[8] S. Hey, „Berührungslose Anregung und Analyse von Schwingungen des menschlichen Auges zur Frühdiagnose einer Glaukomerkrankung,“ 2003. 